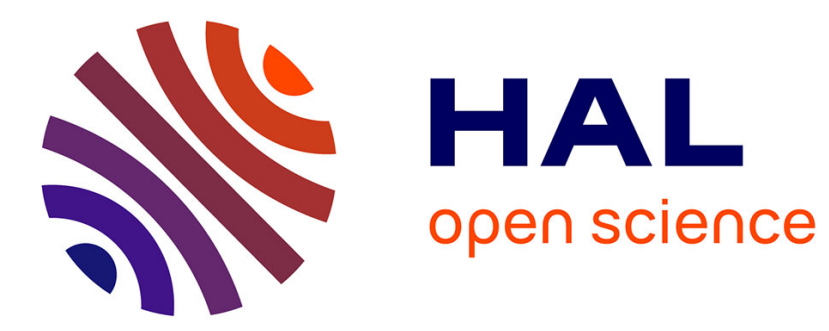

\title{
Naissance des monstres. Le mal et ses doubles dans Britannicus
}

\author{
Tony Gheeraert
}

\section{To cite this version:}

Tony Gheeraert. Naissance des monstres. Le mal et ses doubles dans Britannicus. Racine's Britannicus. A one-day symposium to mark the 350th anniversary of the first performance., Paul Hammond, Sep 2019, Londres, Royaume-Uni. hal-02379296

\section{HAL Id: hal-02379296 https://hal.science/hal-02379296}

Submitted on 25 Nov 2019

HAL is a multi-disciplinary open access archive for the deposit and dissemination of scientific research documents, whether they are published or not. The documents may come from teaching and research institutions in France or abroad, or from public or private research centers.
L'archive ouverte pluridisciplinaire HAL, est destinée au dépôt et à la diffusion de documents scientifiques de niveau recherche, publiés ou non, émanant des établissements d'enseignement et de recherche français ou étrangers, des laboratoires publics ou privés. 


\section{Naissance des monstres. Le mal et ses doubles dans Britannicus}

Prof. Tony Gheeraert, université de Rouen-Normandie

Racine explique, dans sa préface, que Britannicus met en scène une effrayante tératogénèse : face à l'irrésistible révélation du "monstre naissant"1, les autres personnages apparaissent en effet d'abord comme des victimes désemparées du tyran. Le couple d'amoureux innocents, le stoïque conseiller, la mère bafouée même, malgré son "orgueil" et sa "férocité" ${ }^{2}$, tous subissent, impuissants, les premiers coups de la cruauté impériale, aiguisée par les pernicieux conseils de Narcisse. L'évidence de cette lecture binaire s'est d'autant plus aisément imposée que le dramaturge, dans le paratexte, suggère aussi bien la sagesse de Junie ${ }^{3}$, que la "franchise" de Britannicus ${ }^{4}$, ou l'honnêteté de Burrhus ${ }^{5}$. Dans Britannicus, comme dans les autres pièces de Racine, "la vertu et l'innocence surabondent", explique Philippe Sellier, qui voit volontiers se répéter d'une tragédie à l'autre le même scénario obsédant : une nuée d'innocents tourmentée par une poignée de criminels ${ }^{6}$. Georges Forestier, de même, voit dans Britannicus et Junie "un couple de victimes, dont la pureté et l'innocence permettaient de faire ressortir la noirceur du tyran"7, tandis que Burrhus est exemplaire et "vertueux". Britannicus, certes, n'est pas un de ces héros parfaits que chérissait Corneille, mais sa seule faiblesse, aux yeux de l'éditeur de Racine en Pléiade, consiste en sa jeunesse, qui l'empêche de mener une résistance héroïque face au despote. Racine, soucieux de persévérer "dans la voie de ces personnages doubles" d'opposer au monstre naissant un héros lui-même encore imparfait, se serait-il contenté d'un défaut aussi mince, extérieur et conjoncturel ? Nous proposerons ici d'interroger de plus près la vertu supposée du prince, ainsi que de celle de Junie et Burrhus, afin de nous demander si quelque tare cachée ne rendrait pas ces personnages davantage conformes aux principes même de la tragédie, qui exige des héros mêlés. Dans quelle mesure les personnages a priori sympathiques, avec lesquels le public tend spontanément à s'identifier, et dont Racine célèbre la vertu dans la préface, ne sont-ils pas également marqués par le mal, soit qu'ils manifestent eux aussi un penchant naturel vers la perversité, soit qu'ils soient contaminés par le vice environnant ? Au delà d'une approche strictement dramaturgique, il s'agira de se

\footnotetext{
1 Jean Racine, Théâtre-Poésie, édition établie, présentée et annotée par Georges Forestier, Paris, Gallimard, Bibliothèque de la Pléiade, 1999, première préface, p. 372.

${ }^{2}$ Seconde préface, ibid., p. 444.

${ }^{3}$ Première préface, ibid., p. 373.

${ }^{4}$ Ibid.

${ }^{5}$ Seconde préface, ibid., p. 444.

${ }^{6}$ Philippe Sellier, "Le jansénisme des tragédies de Racine : réalités ou illusion ?", in Cahiers de l'Association internationale des études francaises, 1979, 31, p. 135-148 (p. 140). Voir aussi, du même auteur, son introduction au Théâtre complet de Racine, Paris, Imprimerie nationale, t. I, 1995.

7 "Britannicus n'est qu'une pure victime", explique également G. Forestier, in Jean Racine, Théâtre-Poésie, op. cit., p. 1417.

${ }^{8}$ Ibid., p. 1401.
} 
demander si cette peinture d'êtres humains à la moralité ambiguë ne s'accorde pas aussi avec une certaine anthropologie augustinienne, d'autant plus sombre que l'intrigue, inscrite dans l'Antiquité profane, se trouve privée de l'horizon de la Révélation.

\section{Néron et son double}

Britannicus, tout d'abord, est présenté par Racine sous un jour favorable : jeune homme plein de coeur, d'amour et de franchise, spolié injustement de son droit à l'empire, privé de la consolation d'un amour partagé, enfin traîtreusement mis à mort par un apprenti tyran qui fit sur lui l'essai de sa carrière, le tout jeune prince inspire à coup sûr la "compassion". Chacun, en effet, le plaint, et semble des yeux approuver le courroux du fils de Claude. L'antagonisme entre les deux personnages principaux est le principe essentiel de l'action. Le caractère du prince apparaît comme le négatif de celui de son rival : son imprudence spontanée et impulsive s'oppose à la dissimulation cauteleuse et contrôlée de l'empereur. Mais le prince innocent est en même temps "lié [...] par les liens du sang et de l'amitié" 10 à son frère adoptif Néron : la querelle dans les alliances est, comme l'ont montré Aristote et Corneille, le moteur le plus efficace de l'intrigue tragique. Cette fraternité, fût-elle le fruit des manigances d'Agrippine, est soulignée de plus en plus fortement au fur et à mesure que l'action progresse ${ }^{11}$. C'est dire que pour être ennemis, Néron et Britannicus n'en sont pas moins frères, et qu'une secrète ressemblance peut les unir, comme l'avers et le revers de la même médaille.

Or, à y regarder de près, le pitoyable Britannicus partage avec son terrible faux-frère quelques traits de personnalité troublants. Ainsi, son amour pour Junie, loin d'être un sentiment généreux et désintéressé, se définit avant tout comme un désir et un plaisir d'être aimé (V, 1, v. 1504 : "vous m'aimez ?"). Il entretient avec sa maîtresse un rapport de possession, et c'est comme un bien dont on l'a spolié qu'elle apparaît d'abord dans son discours ("Tout ce que j'ai perdu", I, 3, v. 290). Quelques vers plus bas, il considère de nouveau le rapt de Junie comme un larcin dont il serait la victime : "on me l'enlève". Il témoigne aisément du dépit et de la jalousie, et manifeste à l'égard de la princesse une défiance qui perdure jusqu'à leur ultime conversation, où il refuse d'écouter les mises en garde de son amie ( $\mathrm{V}, 1, \mathrm{v} .1535-1543)$. On le voit fugitivement jouir des larmes de sa bien-aimée, dans lesquelles il voit le signe d'une véritable affection ( $\mathrm{v}$. 1555 et 1562) : c'était le rêve de Néron de voir couler de tels pleurs (II, 3, v. 659). La scène des retrouvailles avec Junie (II, 6), alors que Néron est caché, nous fait voir Britannicus sous un jour encore plus troublant. D'abord, à cette occasion, et sous les dehors d'un vocabulaire galant, le prince manifeste moins son amour sincère que son amour-propre: il s'inquiète d'abord de son propre "bonheur" (v. 693 et v. 698), voire de son propre "plaisir" (v. 695), et ne songe qu'à sa "jouissance" (v. 694), dans cette tirade où domine la première personne du singulier. Néron n'est ainsi pas le seul à prétendre

\footnotetext{
${ }^{9}$ Première préface, in Jean Racine, Théâtre-Poésie, op. cit., p. 373.

10 "Fragments du premier livre de la Poétique d'Aristote", in Jean Racine, OEuvres complètes, éd. Pierre Clarac, Paris, Aux Éditions du Seuil, 1962, p. 588.

${ }^{11}$ II, 1, v. 364 ; III, 8, v. 1070 ; IV, 2, v. 1211 et 1217 ; IV, 3, v. 1385 ; V, 5, v. 1626 et v. 1628.
} 
jouir. Au lieu de s'inquiéter de l'état de sa bien-aimée, Britannicus lui reproche en vrai goujat les douleurs que son enlèvement lui cause (v. 706), et de n'avoir pas songé à lui au cours du rapt. L'amour de Britannicus n'est pas seulement égoïste : il y entre une composante sadique, lorsqu'il désire les plaintes de sa maîtresse (v. 704). Enfin, Britannicus laisse affleurer le fantasme d'une autre scène où il serait mort héroïquement aux yeux de son amante.

Égoïste et jouisseur, sadique et possessif, hanté par la théâtralité, poursuivi par les fantasmes : dans ce portrait de Britannicus on reconnaît, en mode mineur, les traits de Néron. La scène répond à celle du récit de l'enlèvement par l'empereur. D'inattendus parallèles se dessinent : comme Britannicus, Néron voile sa perversité sous le masque de l'amour galant. Son comportement et ses propos apparaissent en grande partie déterminés par un amour-propre mal dissimulé sous les oripeaux d'un discours héroïco-précieux. Barthes tentait autrefois d'opposer chez Racine deux amours : "l'Eros sororal" et "l'Eros événement"12. En réalité, chez Racine, comme chez Guilleragues ou Mme de Lafayette, il n'est qu'une espèce d'amour, toujours "chose incommode", toujours possession égoïste, irrépressible, incompatible avec la pureté et la générosité, toujours autodestructrice et vouée à l'échec, à la malédiction et à la souffrance.

L'anthropologie des moralistes, et les cheminements retors de l'amor sui, font apparaître Britannicus comme un double potentiel de Néron. Cette virtualité du mal n'est pas surprenante, au regard des principes même de la poétique : si le jeune prince n'était qu'un "homme vertueux qui tombe du bonheur dans le malheur", il ne serait pas propre à susciter les deux grandes passions tragiques, explique Racine dans sa traduction d'Aristote ${ }^{13}$. A défaut de provoquer la terreur, il faut bien qu'une ombre d'imperfection ternisse sa vertu. Au plan dramatique, Britannicus est une proie, mais ce sont les intrigues d'Agrippine, et non leurs dispositions naturelles, qui ont déterminé les deux "frères" à tenir l'un le rôle de la victime, l'autre celui du bourreau (III, 3, v. 1037-1042 et IV, 2, v. 1151). Sa jeunesse a permis au prince de mourir innocent. Mais que fut devenu ce fils de Messaline, poursuivi par une généalogie aussi redoutable que celle de Néron, s'il avait pu vivre et régner, lui qui ne dispose d'aucune des qualités requises pour gouverner? Trop confiant, incapable de dominer ses passions en présence de l'empereur, n'écoutant que les mauvais conseillers, défiant envers celle-là seule qui pourrait l'aider, tombant aisément dans les pièges qui lui sont tendus: il donne tous les signes d'un futur monstre possible ; le spectateur se souvient que Caligula, et Néron lui-même, ont longtemps su dissimuler [leurs] vices ${ }^{14}$ sous une apparence de vertu. Britannicus n'attire la sympathie que parce qu'il est jeune et vaincu : les aléas de la politique auraient pu changer la donne (III, 8, v. 1037-1040), et inverser les rôles. Comme le dira le choeur d'Antigone chez Anouilh: "Ce n'est pas parce qu'il y en a un qui tue et l'autre qui est tué. C'est une question de distribution...".

\footnotetext{
${ }^{12}$ Roland Barthes, "L'Eros racinien", in Esprit, 278/11, nov. 1959, p. 471-482. Repris dans "Les deux Eros", Sur Racine, Paris, Le Seuil, 1963.

13 "Fragments du premier livre de la Poétique d'Aristote", in Jean Racine, Oeuvres complètes, op. cit., p. 588.

${ }^{14}$ Préface de 1675, in Théâtre-poésie, op. cit., p. 444.
} 
Ou plutôt, pour l'ancien élève de Port-Royal, c'est une question de nature humaine. Britannicus ne saurait être sans faiblesse, non seulement pour des raisons d'ordre dramaturgique, mais aussi anthropologiques, voire théologiques.

\section{Narcisse et son double}

"Il ne faut pas regarder Burrhus comme un homme vertueux absolument. II ne l'est qu'en opposition avec Narcisse" (Chamfort).

Le coeur de Britannicus comporte ainsi quelques germes mauvais que sa prompte mort ne donnera pas l'occasion de laisser s'épanouir. Mais Burrhus, le conseiller du prince, n'offre-t-il pas le visage d'une vertu admirable et sans mélange ? Racine nous invite à révérer la figure du vieux gouverneur, qu'il a choisi de préférence à Sénèque en raison de "la sévérité de ses moeurs"15. Rares sont les voix à avoir sérieusement discuté cette image avantageuse. G. Forestier, qui admet aisément la "duplicité" des deux principaux protagonistes, considère plus uniment Burrhus et Sénèque comme "deux conseillers vertueux"16. Selon lui, Burrhus est "mû par la seule vertu", et si le gouverneur est conduit à l'échec, c'est seulement parce qu'il se trouve face à un tyran que sa situation politique rend inaccessible à une conversion morale : il n'y a "ni légitimité, ni vertu possible pour un usurpateur" ${ }^{17}$.

Burrhus, en apparence, appartient ainsi à cette race des vieux Romains incorruptibles que Corneille avait souvent mis sur scène. Vaillant légionnaire un peu fruste, il affirme son entière sincérité (I, 2, v. 141) et revendique ainsi la franchise d'une parrêsia qui est le propre de l'homme honnête (I, 2, v. 174), mais aussi, tout particulièrement, du stoïcien. L'ami de Sénèque partage en effet les vues philosophiques de ce dernier, et apparaît comme un double du Cordouan $^{18}$, ainsi que la critique récente l'a définitivement établi. L'idéal éthico-politique de Burrhus ne manque pas de noblesse : il espère que Néron sera guidé par le Bien, le sens du devoir, le souci de ses peuples, de leur bonheur et de leur liberté (I, 2, v. 200-202 ; IV, 3, v. 1337-1338) ${ }^{19}$. Austère, intègre, exigeant, Burrhus est ainsi conforme à l'image donnée de lui par les Anciens, et souvent relayée au XVIle siècle.

\footnotetext{
${ }^{15}$ Préface de 1675, in Théâtre-Poésie, op. cit., p. 444.

${ }^{16}$ Georges Forestier, Jean Racine, Paris, Gallimard, NRF Biographies, 2006, p. 369.

17 Théâtre-Poésie, op. cit., p. 1409-1410.

18 "Racine prête les paroles de Sénèque à Burrhus", déclare Volker Schröder, La tragédie du sang d'Auguste. Politique et intertextualité dans Britannicus, Tûbingen, G. Narr, second tirage, 2004, p. 132. Voir tout le chapitre : "Sénèque : le miroir du prince", p. 123-141. Voir aussi Katsuya Nagamori, "Burrhus... assume ici, en l'absence de Sénèque, le rôle du sage stoïcien", in "Racine et Sénèque. L'échec d'un idéal stoïcien dans la tragédie racinienne », Dix-septième siècle, vol. 248 , no. 3,2010 , p. 431-441.

19 "Ce rêve d'une union idéale entre principat, liberté et vertu me semble constituer le coeur de la philosophie politico-morale de Burrhus, ainsi que la justification théorique de son propre rôle de gouverneur", écrit ainsi V. Schröder, La Tragédie du sang d'Auguste, op. cit., p. 123.
} 
Quelques critiques soupçonneux se sont demandés toutefois si cette vertu n'était pas trop parfaite pour n'être pas suspecte ${ }^{20}$. Jean Rohou, qui suspecte l'amour-propre du gouverneur, ne cautionne pas les insinuations de duplicité, de mensonge et d'ambition lancées par Narcisse (IV, 4, v. 1461-1462). II est vrai que, à plusieurs reprises, et malgré sa carapace de vertu, nous voyons Burrhus transiger avec ses principes. Ainsi, nous savons qu'il réprouve l'enlèvement de Junie (III, 2), et il fait savoir à Néron qu'il condamne cette injustice (III,1), mais il tâche néanmoins de disculper l'empereur devant Agrippine en exposant les raisons politiques de cette arrestation (I, 2, v. 235-244). Délicat porte-à-faux, voire flagrant délit de double discours, de la part d'un philosophe affectant l'éthos d'un parrèsiaste qui sait "mal farder la vérité" (I, 1, v. 174). Plus loin, Burrhus tient des propos au moins ambigus, à défaut d'être tout à fait mensongers, lorsqu'il soutient devant l'empereur que Britannicus est innocent des complots dont on l'accuse (IV, 3, v. 1386-1387), alors même que le jeune prince a tenté, certes vainement, d'activer ses soutiens parmi la noblesse sénatoriale. De plus, grisé par l'importance que lui donne son poste de " gouverneur ", et l'ascendant qu'il pense encore exercer sur son élève, Burrhus indispose Agrippine en la ravalant au rang du « public » qui ignore les dédales du palais (I, 2, v. 135): Burrhus manifeste ici un vain goût pour une gloire de mauvais aloi. En courtisan, il guette l'oreille du prince qui "l'écoute encor" (v. 689). Longtemps, il jettera sur l'empereur le regard d'un Pygmalion satisfait contemplant sa Galathée, heureux de voir son disciple obéir aux principes qu'il croit lui avoir inculqués : il le considère comme son "ouvrage", ainsi que le souligne Agrippine (I, 2, v. 223).

Burrhus comme Narcisse se ressemblent autant qu'ils s'opposent : tous deux rêvent d'un empereur modelé selon leur désirs. Burrhus, drapé du costume de philosophe vertueux, et Narcisse, la "peste de cour ", déploient des stratégies qui ne sont différentes qu'en apparence : ils aspirent tous deux à contrôler Néron et à en faire leur "ouvrage". Autant que son antithèse, Burrhus apparaît comme un double de Narcisse, habité comme lui par la libido dominandi. A lire les textes de l'époque, les jugements des commentateurs étaient plus mitigés, et ne donnaient pas de Burrhus l'image d'une aussi parfaite vertu que celle alléguée par Racine dans ses préfaces. En 1622, le juriste Didier Hérauld, dans son Fragment de l'examen du Prince de Machiavel, donne du gouverneur de Néron une image mêlée : leur mort, comme l'écrasement du Sénat, sont la juste récompense de leur lâcheté et de leur complicité avec l'empereur, en particulier à l'occasion de la répudiation et de l'exécution d'Octavie.

Dira-t-on que Burrhus, ce grand Stoïque [...] pour s'être accommodé à lui en un si détestable acte, aient rompu le cour de ces cruautés ? Tout au contraire, ils en ont expérimenté eux-mêmes les justes effets.... Et de fait, Burrhus et Sénèque en échappèrent-ils [à la mort] pour avoir non seulement connivé au parricide de Néron,

20 Voir en particulier Jean Rohou, "Les complaisances du vertueux Burrhus", in Racine/Britannicus, éd. Pierre Ronzeaud, Paris, Klincksieck, 1995, p. 163-175. Pour un bilan des jugements contradictoires sur Burrhus, voir Volker Schröder, La Tragédie du sang d'Auguste, op. cit., p. 106-107. 
mais même y avoir participé ? Ils furent salariés comme les autres, et à la vérité ils le méritaient bien. ${ }^{21}$

L'implication de Sénèque, en particulier, a entièrement "noirci et effacé ses autres vertus", mais Hérauld est également sévère avec le tribun. De même Balzac, qui propose un portrait d'abord avantageux du ministre, puis plus mitigée ${ }^{22}$, entaché par trois griefs : d'abord Burrhus, bien que "sans reproche", a dû se compromettre pour rester en place : sa "vertu [...] se savait quelquefois accommoder à la corruption de son temps, et [...] souffrait ce qu'elle n'approuvait pas." ${ }^{23}$ Ensuite, Burrhus cautionnait par sa présence et sa sagesse même le règne illégitime d'un usurpateur (Sénèque et Burrhus "autorisaient par leur présence, rendaient en quelque façon légitime, une principauté mal acquise"24). Enfin, selon Balzac, Burrhus a imprudemment joué les apprentis-sorciers, et son échec était inévitable : "le démon étrangle à la fin le magicien qui pensait le gouverner"25.

On est moins surpris, à lire les jugements mêlés portés par les contemporains sur Burrhus, de rencontrer quelques failles dans l'armure de vertu que revêt le vieux soldat. Agripppine, reprenant en quelque sorte les arguments d'Hérauld et Balzac, ne se fait pas faute de souligner la complicité de Burrhus, au moins passive, dans tous les forfaits qu'elle a dû perpétrer : elle l'associe à ses "crimes" ("nos crimes communs", III, 3 , v. 849). La première faute de Burrhus est d'avoir accepté la proposition d'Agrippine : "l'ambition", qui a poussé le vieux soldat à quitter "les honneurs obscurs de quelque légion" (I, 2, v. 154), l'a précipité dans un tourbillon où il a travaillé en vain au bonheur public, car à fréquenter le monde, on y perd nécessairement son âme ${ }^{26}$. "Le Portique" est incompatible avec "la cour", pour reprendre le titre d'une étude que Normand Doiron avait consacré à ce sujet ${ }^{27}$.

Le gouverneur, qui tient les discours stoïciens de Sénèque, tombe dans tous les travers du stoïcisme recensés par les augustiniens de Port-Royal; larmoyant, incapable de maîtriser ses émotions, il n'est pas jusqu'à son appel à la clémence qui ne soit suspect: les philosophes du Portique avaient érigé en vertu cette clementia, comme signe de magnanimité du souverain, utile à l'équilibre public. Dans son traité de la Clémence adressé à Néron, et dont Burrhus reprend ici un argument, Sénèque expliquait que cette vertu avait pour but d' "attirer l'attention publique" et d'assurer au

\footnotetext{
${ }^{21}$ Didier Hérauld, Fragment de l'examen du Prince de Machiavel, Paris, Abraham Picard, 1622, p. 104-108.

${ }^{22}$ Les Oeuvres diverses du sieur de Balzac, Amsterdam, Elzevier, 1664, p. 293 et suivantes.

${ }^{23}$ Ibid., p. 294.

${ }^{24}$ Ibid., p. 296.

25 Ibid.

26 Dans Arie et Petus de Gilbert (1660), Petus reprochait amèrement à Sénèque sa compromission à la cour : "Tu fais trop bien ta cour pour un sage Stoïque. De l'amour des grandeurs indignement épris, ta vertu répond mal à tes divins écrits : tes discours généreux ne sont que du langage, et pour m'expliquer mieux, Néron est ton ouvrage... $(I, 5)$

${ }^{27}$ Normand Doiron, « Le Portique et la cour. Néo-stoïcisme et théorie de l'honnêteté au XVIle siècle », Dix-septième siècle, vol. 213, no. 4, 2001, p. 689-698.
} 
prince réputation et renommée: c'était mettre en avant une conception utilitaire de la vertu, fondée sur l'intérêt. C'est cette qualité dévoyée, parce que fondée sur l'amour-propre, que Burrhus propose à Néron pour l'empêcher de tomber dans le gouffre du crime:

Quel plaisir de penser et de dire en vous-même :

«Partout, en ce moment, on me bénit, on m'aime ;

On ne voit point le peuple à mon nom s'alarmer ;

Le ciel dans tous leurs pleurs ne m'entend point nommer ;

Leur sombre inimitié ne fuit point mon visage ;

Je vois voler partout les cœurs à mon passage ! » (IV, 3, v. 1360-1364)

Pour préserver l'empereur de basculer dans le mal, Burrhus ne trouve d'autre ressource que de "flatter son amour-propre", ainsi que le remarquait déjà Louis Racine 28 : loin de proposer à Néron de suivre la vertu comme un absolu, il la réduit à un instrument de "plaisir". Cet argumentaire pose plusieurs difficultés. D'abord, ce discours, inspiré du De Clementia de Sénèque, fait éclater les contradictions internes du système stoïcien, puisque le philosophe espagnol affirme par ailleurs que le bien moral constitue en lui-même sa propre finalité ${ }^{29}$; la vertu de la tirade citée à l'acte IV est donc très douteuse, et de plus fragile et inefficace : l'image idéalisante de lui-même que Burrhus propose à Néron ne résistera pas longtemps aux plus séduisantes tentations que lui suggérera Narcisse dans la scène suivante. La tirade de Burrhus illustre l'incohérence de la vertu stoïcienne aux yeux des augustiniens : elle n'est que le masque et l'instrument de l'amour-propre. Faute d'avoir été initié aux vérités du christianisme interprété selon les lumières de saint Augustin, Burrhus ignore la distinction fondatrice entre usage et jouissance : "Nous disons donc que nous jouissons à propos de ce dont nous tirons plaisir. Nous usons de ce que nous référons à ce dont il s'agit de tirer plaisir"30. L'auteur de la Cité de Dieu, suivi sur ce point par Pascal et Port-Royal, y critique la fausse vertu des païens qui les conduit à jouir d'eux-mêmes et de leur propre vertu apparente ${ }^{31}$. Or, Burrhus propose précisément à Néron de se faire aimer de son peuple grâce à sa clémence. Autrement dit, le tribun suggère à l'empereur d'user de la vertu afin de jouir de lui-même à travers le reflet embellissant que lui renverra le peuple. Or, une telle vertu prétendue n'est qu'une insupportable idolâtrie : elle n'est pas pratiquée en vue de Dieu, mais pour l'amour de soi même ; elle réduit le prochain au rang de simple instrument sa propre vanité ${ }^{32}$.

\footnotetext{
${ }^{28}$ Louis Racine, Remarques sur Britannicus, cité par Volker Schröder, op. cit., p. 126.

29 "Le profit authentique de nos bonnes actions consiste à les avoir faites, et il n'y a point de digne récompense à nos vertus en-dehors d'elle-même", Sénèque, De la Clémence, I, 1.

${ }^{30}$ Saint Augustin, Quatre-vingt-trois questions diverses, question 30.

${ }^{31}$ Voir Philippe Sellier, Pascal et saint Augustin, Paris, Albin Michel, 1995, p. 152 sqq.

${ }^{32}$ Sur la distinction uti et frui chez saint Augustin et à Port-Royal, voir Philippe Sellier, Pascal et saint Augustin, op. cit., p. 158. Et Isabelle Koch, "Augustin et l'usage du monde ", Cahiers philosophiques, vol. $122, n^{\circ} 2,2010$, p. 21-42. On trouvera dans ces deux travaux une analyse précise de la critique augustinienne des fausses vertus des païens.
} 
Les disciples d'Augustin voyaient encore dans la clémence une autre tare : elle constitue à leurs yeux un inique déni de justice, voire une lâcheté, ainsi que l'expliquera bientôt Jacques Esprit, moraliste proche de Port-Royal ${ }^{33}$. Aussi, la répugnance de Néron à signer un acte de condamnation, dans laquelle Burrhus veut lire un signe signe de bon naturel, peut-elle être aussi interprétée comme une première entorse à un idéal de justice qui implique que chacun reçoive ce qu'il mérite.

Volker Schröder se refuse à juger de la moralité de Burrhus à partir de cet appel à la clémence : il n'y voir qu'une stratégie rhétorique pour persuader Néron, et souligne à juste titre la similitude entre les arguments de Burrhus et ceux de Narcisse, qui visent à toucher les passions de l'empereur. Mais quand bien même la stratégie de Burrhus n'aurait en vue que l'efficacité, et quand bien même le tribun n'adhérerait pas aux raisons avancées dans son discours, il n'en serait pas moins coupable, au regard des règles stoïciennes qui régissent l'usage de la parole. Sénèque exige en effet de l'orateur la modération et la maîtrise, afin de ne pas "compromettre sa dignité morale"34 . Burrhus au contraire s'épanche en souvenirs et confidences, pleurniche (v. 1381), laisse percer ses émotions de vieillard. Chantage au suicide (v. 1374-1375), démonstrations physiques soulignées par une didascalie (v. 1377) : Burrhus recourt à toute une véhémence passionnelle que Sénèque interdit et abandonne au "déclamateur ambulant", exigeant plus de retenue et de grandeur chez le stoïcien ${ }^{35}$.

Burrhus ne se contente pas de promouvoir un principe douteux comme la clémence, l'une des plus redoutables parmi les fausses vertus des païens : à plus d'une reprise, le gouverneur est saisi en flagrant délit d'infraction à l'encontre des principes mêmes du stoïcisme qu'il représente en l'absence de Sénèque. Loin de garder en toutes circonstances l'impassibilité du Sage, il pleure, non seulement devant Néron, mais aussi devant Agrippine ( $V, 5$, v. 1654). De même, il renonce aisément aux velléités de partir en exil ( $V, 5$, v. 1625) ; il se garde également de donner suite aux serments emphatiques d'attenter à ses jours : (IV, 3, v. 1373-1376 et V, 5, v. 1624): au lieu d'opter pour ce trépas qui eût été conforme à celui des grands modèles de sa secte on songe par exemple à Caton d'Utique -, il se contente d'espérer une improbable rédemption de son pupille ( $\mathrm{V}$, scène dernière, $v$. 1788). La reculade du gouverneur ne surprend pas le lecteur des Essais de morale : Nicole nous avait bien prévenu que ces philosophes n'étaient que des matamores de la vertu (ou plutôt des « fanfarons de

33 Voir Guillaume Flamerie de Lachapelle, "Jacques Esprit, Sénèque et la clémence ", Anabases, 4, 2006, 111-123, en ligne. "Esprit adopte différentes attitudes face aux arguments païens et sénéquiens. II estime que certains sont justes, mais que le Stoïcien n'en tire pas toutes les conséquences, ce qui invalide la thèse finale de ce dernier et prouve son incohérence ; que d'autres travestissent une sombre réalité sous des dehors flatteurs, et doivent être dénoncés ; que d'autres enfin sont tout à fait contraires à la vérité. Ces réfutations n'excluent pas les blâmes dirigés contre la personne même de Sénèque.", $\S 5$.

${ }^{34}$ Lettres à Lucilius, 40.

${ }^{35}$ Lettres à Lucilius, 40 ; voir aussi la lettre 75 : "l'éloquence qui se consacre à la vérité doit être simple et sans apprêt... Voici le point essentiel de notre intention. Dire ce que l'on pense, penser ce que l'on dit ; faire que le langage soit d'accord avec la conduite. II a rempli ses engagements, celui qui, à le voir et à l'écouter, se trouve le même." 
morale ", comme dira bientôt Guéret ${ }^{36}$ ) Les philosophes qui prêchent le suicide aux temps heureux, se déroberont le moment venu : "Mais laissez le faire, il trouvera bien moyen de s'exempter de mourir, il n'en trouvera jamais d'occasion”. C'est précisément ce qui arrive au dénouement : Burrhus "s'exempte de mourir". Bien loin de finir sa vie, Burrhus va continuer à déchoir : les lecteurs informés de l'histoire romaine savent que la réputation de Burrhus se trouve ternie après la mort de Britannicus, dont il acceptera les biens ${ }^{37}$.

Enfin, Burrhus manque au fatalisme stoïcien : Le Stoïcien doit "vouloir que les choses arrivent comme elles arrivent", estime Epictète ${ }^{38}$. Burrhus, lui, croit, jusqu'au bout, en une possibilité d'agir sur le cours des choses, et ne manifeste guère la soumission à la Providence prônée par la secte du Portique. Aux yeux des Stoïciens, la chaîne des événements, fût-elle funeste aux yeux des hommes, s'inscrit dans un ordre du monde gouverné par la Raison, et auquel il convient donc d'acquiescer ; mais la vertu de Burrhus ne saurait aller si loin. Le seul personnage à se réclamer du Destin, c'est Néron : l'élève indocile retourne contre son frère et sa mère un argument qu'il a souvent dû entendre dans la bouche de ses précepteurs, et qu'il dévoie afin d'éluder ses responsabilités (III, 8, v. 1041 ; V, 7, v. 1676).

Tout le comportement de Burrhus apparaît ainsi comme l'illustration des critiques anti-stoïciennes émanant des milieux augustiniens, et en particulier de Port-Royal. Le tragique du personnage procède précisément du décalage entre d'une part ses aspirations politico-éthiques, et de l'autre son incapacité à y parvenir, en raison non seulement des faiblesses de son élève, évidentes, mais aussi des siennes propres. Le sage n'est pas capable de se hisser à la hauteur de l'idéal qu'il professe.

Burrhus pouvait paraître d'une vertu monolithique. En réalité, il est déchiré, et c'est cette fracture qui constitue son humanité tragique : il est tout ensemble grand et misérable. D'autant plus tragique qu'il ne se connaît pas misérable, obnubilé par son rêve et par ses illusions. Burrhus fait éclater malgré lui les contradictions et les inconséquences de la philosophie qu'il professe, et que mettent en évidence Pascal, dans l'Entretien avec M. de Sacy ${ }^{39}$, ou Pierre Nicole dans les Essais de morale ${ }^{40}$. Tous deux reprochent à Epictète et Sénèque leur présomption insensée, et leur prétention à détenir une sagesse qui n'est en réalité que folie. Ils reprochent aux Stoïciens de prêcher une morale de résistance aux passions qui pourrait à la rigueur apparaître louable dans ses finalités, mais est en réalité impraticable étant donné l'état de

\footnotetext{
${ }^{36}$ Gabriel Guéret, La Guerre des auteurs anciens et modernes, Paris, Théodore Girard, 1671, p. 28-30.

${ }^{37}$ Tacite, Annales, XIII, 18.

${ }^{38}$ Entretiens, I, 35.

39 "Entretien avec M. de Sacy", in B. Pscal, Oeuvres complètes, préface d'Henri Gouhier, présentation et notes de Louis Lafuma, Paris, Aux Éditions du Seuil, 1963, p. 291-297.

${ }_{40}$ Pierre Nicole, "De l'éducation d'un prince. Troisième traité [...] Réflexion sur le livre de Sénèque De la Brièveté de la vie", in Essais de morale. volume second, Paris, Guillaume Desprez, 1682.
} 
l'homme pécheur ${ }^{41}$. Le sage stoïcien n'est qu'un orgueilleux qui se méprend sur la nature humaine : telle est bien la faute tragique de Burrhus, l'hybris à laquelle il succombe, la démesure qui le rend fautif, quelque nobles que soient ses ambitions. Certes, le vieux soldat n'est pas tout à fait coupable, puisqu'il ne commet pas le mal délibérément, et qu'il cherche au contraire sincèrement à travailler au bien public; mais il n'est pas non plus tout à fait innocent : naïf à force d'orgueil et de vanité, il ne parvient pas à élever son élève jusqu'à son idéal, mais lui-même n'y atteint pas non plus. On aurait tort d'attribuer l'insuccès du gouverneur au seul coeur rétif de son élève, trop veule pour faire preuve de cette volonté qu'exige de lui son maître : c'est que l'effort exigé par Burrhus est irréaliste. Demander à Néron de renoncer à Junie à force de volonté ("On n'aime point si l'on ne veut aimer"), c'est trop exiger, et plus que l'être humain ne peut tenir. L'espérance chimérique du gouverneur illustre l'opinion de Nicole sur les stoïciens : "Les philosophes commandaient l'impossible"42. Burrhus est un mauvais éducateur parce qu'il ne fonde pas son enseignement sur une juste connaissance du coeur : à ce titre, il porte une part de responsabilité dans le naufrage pédagogique que constitue aussi cette funeste journée. II commet une erreur dénoncée par Nicole : "de croire que I'homme se conduise par raison, au lieu qu'il ne se conduit que par passion". La tension de la volonté qu'il cherche à imposer à Néron, de même que l'ataraxie qu'il lui propose en guise de bonheur ("le repos", v. 1358) voleront en éclats devant les promesses de plaisir immédiat offertes par Narcisse, plus fin connaisseur que Burrhus des ressorts de l'âme humaine.

L'échec de Burrhus, comme celui des stoïciens en général, c'est de n'avoir pas "bien connu l'homme", ainsi que l'explique Pascal. Ce n'est pas la duplicité ni l'hypocrisie que reprochent aux Stoïciens les augustiniens de Port-Royal : c'est leur aveuglement sur les autres et sur eux-mêmes. Ils mentent, car ils se mentent, par ignorance de la vraie nature pécheresse de l'homme.

Ils ne se disent jamais vrai à eux-mêmes. Sénèque connaissait l'aveuglement des Grands ; mais il ne connaissait pas l'aveuglement des philolophes, ni le sien. C'est qu'il ne connaissait pas parfaitement l'aveuglement même des Grands. Pour le bien connaître il faut pénétrer, non seulement l'aveuglement attaché à certains états; mais aussi l'aveuglement général de l'homme. ${ }^{43}$

Burrhus, de même, ne connaît qu'une moitié de l'être humain, sa grandeur, et il en ignore la misère. Epictète, dit Pascal, "mériterait d'être adoré... s'il avait connu son impuissance... après avoir si bien compris ce qu'on doit, voici comment [Epictète] se perd dans la présomption de ce qu'on peut"44.

\footnotetext{
${ }^{41}$ Voir là encore le commentaire de Jacques Esprit par Guillaume Flamerie de Lachapelle, art. cit.

${ }^{42}$ Réflexion sur le livre de Sénèque De la Brièveté de la vie, op. cit., p. 72.

${ }^{43}$ Ibid., p. 82.

${ }^{44}$ Blaise Pascal, "Entretien avec M. de Sacy", op. cit., p. 293.
} 
En définitive, sous couleur de vertu, c'est un portrait sévère que Racine nous brosse de Burrhus. Certes, comme le notait déjà Jean Rohou, on ne peut soupçonner le gouverneur de nulle duplicité consciente ${ }^{45}$. Mais son acceptation courtisane des compromissions, son espoir vain de travailler à l'établissement d'une société plus juste, ou encore une forme de complexe de Pygmalion, viennent donner de la profondeur à un personnage qu'on aurait cru tout d'une pièce. Si le gouverneur austère et rigoureux n'échappe pas à l'universalité d'un mal insidieux et souterrain, la princesse Junie montrerait-elle la voie d'une vertu véritable?

\section{Junie équivoque}

Lucien Goldmann voyait en Junie la véritable héroïne, incarnation des valeurs "extramondaines", et annonçant de loin Esther ${ }^{46}$. Junie n'occupe pourtant qu'un petit rôle dans Britannicus, et ne manifeste pas la grandeur sombre de Néron ou de sa mère. Elle pourrait même passer pour une utilité dramatique: comme l'a montré Georges Forestier, elle sert d'abord à nouer le fil sentimental de l'histoire au fil politique 47. Ce personnage féminin, au coeur de l'intrigue mais en marge de l'action, échappe-t-il au moins à la noirceur universelle ? La pureté de Junie a été trop commentée pour qu'il soit nécessaire d'y revenir : princesse par sa naissance, mais avant tout amoureuse dépourvue d'ambition, méfiante et lucide sur les dangers de la cour, habitée par le rêve pastoral d'un amour heureux vécu hors de l'agitation du monde, elle préfigure Bérénice. II n'y a pas lieu de douter de la sincérité de son engagement ni de son aspiration à la retraite. C'est Néron qui, à la faveur d'un enlèvement brutal, la contraint à résider au palais. Mais dès qu'elle pénètre dans ce lieu de corruption, elle est perdue, et se trouve aussitôt souillée par un mal qu'elle cherchait à éviter. Non seulement elle est entraînée par des soudards, et exposée aux regards et aux désirs pervers de l'empereur, mais elle subit aussi un avilissement moral qu'elle finit par accepter. Lorsque l'empereur exige qu'elle bannisse celui qu'elle aime, par ses "discours", ses "silences", ou ses "froideurs" (II, 3, v. 672-673), Junie se retrouve en effet contrainte de parler non seulement contre son son amour, mais aussi contre la vérité de son coeur. Lors de sa première entrevue avec le prince (II, 6), face à la franchise de son amant tout de flamme, il lui faut biaiser afin de le sauver malgré lui. Confrontée à un insoluble cas de conscience, elle use d'abord du mensonge : on peut douter en effet que Britannicus ait passé son temps, au cours de ses entretiens amoureux avec Junie, à rendre hommage à son faux-frère ("Toujours à sa vertu vous rendiez quelque hommage", v. 727). Elle pratique également la restriction mentale, en refusant obstinément de répondre aux questions de Britannicus (v. 736, 740, 741). Enfin, elle recourt à l'équivoque : "Ces murs mêmes, Seigneur, peuvent avoir des yeux"

\footnotetext{
45 "Les complaisances du vertueux Burrhus", art. cit., p. 175 : "Burrhus ne se déguise pas volontairement, par ambition, mais par réaction involontaire d'amour-propre".

46 "La fin de Junie indique Esther et Athalie", Lucien Goldmann, Le Dieu caché, Paris, Gallimard, Tel, 1959, p. 368.

47 "Donner à Britannicus une amoureuse, c'était créer un couple de victimes [...] dont les amours contrariées pouvaient donner à la pièce une dimension élégiaque de nature à contrebalancer la tonalité fortement politique de la donnée initiale », Georges Forestier, préface à son édition de Britannicus, Paris, Gallimard (Folio théâtre), 1995, p. 14.
} 
(v. 713) : la figure, au lieu de désigner clairement la présence de Néron, laisse entendre seulement que le palais est rempli d'espions. Mensonges, louanges hypocrites (v. 726), restrictio mentalis et équivoques : on reconnaît les méthodes de la casuistique jésuite $^{48}$. L'équivoque est une ruse linguistique connue depuis longtemps, mais que les jésuites ont systématisée à travers une doctrine exposée en particulier Sanchez et Navarre. L'usage de l'équivoque par Junie pourrait trouver quelque excuse aux yeux des bons Pères, puisqu'elle permet d'éviter la contre-vérité, et sert à protéger un innocent:

Une chose des plus embarrassantes est d'éviter le mensonge, et surtout quand on voudrait faire accroire une chose fausse. C'est à quoi sert admirablement notre doctrine des équivoques, par laquelle il est permis d'user de termes ambigus, en les faisant entendre en un autre sens qu'on ne les entend soi-même. ${ }^{49}$

Mais Port-Royal est plus intransigeant. Pascal, dans les Provinciales, avait traité par la raillerie cinglante la doctrine des équivoques, artifice hypocrite conçu selon lui pour autoriser le mensonge. "Quiconque se sert du mensonge agit par l'esprit du diable", expliquait le polémiste. La falsification du vrai est toujours criminelle, quand bien même l'intention du menteur serait bonne et la finalité louable : " on ne doit pas faire le moindre mal pour en faire réussir le plus grand $\operatorname{bien}^{50}$ ». Le vrai étant un bien absolu, aucun intérêt ne peut lui être jugé supérieur. Boileau, dans la Satire XII verra de même dans l'équivoque chère aux Bons Pères une "fourbe insigne". La sévérité des augustiniens s'appuie sur la prohibition absolue du mensonge imposée par l'évêque d'Hippone dans les deux traités qu'il a consacrés à ce sujet ${ }^{51}$ : selon Augustin, en aucun cas il ne faut falsifier le réel, ni avec des mots, ni avec des signes extra-linguistiques: "Ment donc qui a une chose dans l'esprit et en avance une autre, au moyen de mots ou de n'importe quel autre type de signes"52. C'est ainsi avec raison que Britannicus reprochera à son amie de l'avoir trompé non seulement par ses discours, mais aussi par le silence de ses regards ${ }^{53}$.

En réduisant Junie au mensonge, au silence et à la simulation, Néron est parvenu à ses fins : "irrité" (II, 2, v. 418) par la vertu de Junie comme un papillon de nuit par la lumière, il a enfermé la princesse dans une impasse morale dont elle ne pouvait sortir qu'en acceptant de se compromettre. Elle s'était flattée de sa sincérité : "Toujours de mon coeur ma bouche est l'interprète" (II, 3, v. 640). Présomptueuse affirmation, que

${ }^{48}$ Voir sur ces questions Jean-Pierre Cavaillé, "Histoires d'équivoques ", Les Cahiers du Centre de Recherches Historiques, 33, 2004, en ligne ; Sophie Gouverneur, "Les Provinciales et la doctrine des équivoques à l'épreuve de la politique, XVIle Siècle, 1, 242, 2009, p. 149-158. ${ }^{49}$ Cité par Blaise Pascal dans la 9e Provinciale (Les Provinciales, Paris, Classiques Garnier, 1984, p. 164).

50 11e Provinciale, ibid., p. 264.

${ }^{51}$ Du Mensonge et Contre le mensonge.

${ }^{52}$ Du Mensonge, III, 3, in OEuvres, Paris, Gallimard, Bibliothèque de La Pléiade, 1999, vol. I, p. 734-735.

${ }^{53}$ Sur les restrictions mentales, voir Pierre Cariou, "Des restrictions mentales », in Pascal et la casuistique, sous la direction de Pierre Cariou, Presses Universitaires de France, 1993, p. 133-156. 
Néron la force à démentir. Grâce à son stratagème, Néron jouit certes d'abord du plaisir de voir son rival repoussé ; mais aussi, et surtout, il réussit à corrompre Junie : par amour, dans l'espoir de soustraire son amant au péril, elle sacrifie sa vertu, son innocence, sa franchise ; elle se prête au jeu de rôle théâtral qu'impose son nouveau maître, et, en se pliant à cette mise en scène perverse, elle subit une défloration morale.

Junie, pendant son bref passage à la cour, y perd la virginité de son âme. Le portrait que Racine donne de la cour est conforme à l'image qu'en proposait Port-Royal, et plus particulièrement Arnauld d'Andilly, Solitaire envers qui Racine éprouvait une profonde affection ${ }^{54}$. Dans ses Oeuvres chrétiennes, le poète décrivait la cour en des termes qui préfigurent Britannicus:
Veux-tu voir une scène en merveilles féconde?
Considère la cour ; c'est là qu'à tous moments
Agissent les ressorts de ces grands mouvements
Qui font changer de face au théâtre du monde :
C'est là que tout excelle en l'art des fictions ;
C'est là que l'intérêt règle les passions ;
C'est là que du malheur l'insolence se joue ;
C'est là qu'à la Fortune on dresse des autels ;
Et que l'ambition, pour monter sur sa roue,
Fait les plus grands flatteurs des plus grands des mortels. ${ }^{55}$

Britannicus illustre cette représentation au vitriol d'une cour conçue comme le raccourci des perversions du monde : Agrippine, cachée derrière un voile, préside aux "mouvements" de ce "grand corps" politique, tandis que règnent l'intérêt, les fictions, la mise en scène, la flatterie, et le malheur persécuté par l'insolence. A deux reprises, Narcisse puis Néron évoquent la Fortune dont d'Andilly, favori passé en disgrâce, avait goûté à ses dépens les vicissitudes. Le thème du mépris du monde, certes, n'était pas propre à Port-Royal. Mais cette critique de la cour d'inspiration augustinienne n'était pas non plus générale sur la scène classique : depuis Castiglione, la cour reste aussi l'endroit idéal où devraient pouvoir s'épanouir toutes les qualités morales et physiques ; Molière, trois ans après Britannicus, introduit une apologie de la cour dans les Femmes savantes, tandis que, depuis Faret, de nombreux manuels continuent d'enseigner l'art d'être un bon courtisan. Racine, trois ans après le fâcheux épisodes de la réponse à l'auteur des Imaginaires, se rapproche maintenant de Port-Royal, et retrouve les accents des auteurs proches du monastère ${ }^{56}$. Alors même qu'il se trouve

\footnotetext{
${ }^{54}$ Jean Lesaulnier a produit récemment un témoignage supplémentaire de cette proximité entre le jardinier de Port-Royal et le jeune Racine : dans son édition de la Correspondance de Jean Racine (Paris, Honoré Champion, 2017, p. 655-662, l'éditeur introduit un poème inédit composé par le futur dramaturge à l'occasion d'une convalescence d'Arnauld d'Andilly

${ }^{55}$ Robert Arnauld d'Andilly, Stances sur diverses vérités chrétiennes, Paris, Pierre Le Petit, 1642, stance 199.

56 Sur "la grande réconciliation" entre Racine et Port-Royal, "au tournant des années 1668-1669", voir Georges Forestier, Jean Racine, op. cit., p. 346-348. En 1669, Jean Racine
} 
au milieu du tourbillon mondain, occupé à grimper les degrés d'une brillante carrière, il laisse voir que nul ne peut à la cour garder son innocence, et que seule la solitude confère la paix du coeur, loin $d u$ monde et $d u$ bruit. Face aux tentations et aux menaces du monde, la fuite reste en effet la seule issue. Burrhus, en quelque sorte salésien, a cru pouvoir concilier le siècle et la vertu. Junie, plus conséquente que le gouverneur du prince, sait que la retraite seule la préservera de l'infection qui gangrène le palais impérial. Sitôt arrivée, elle réclame l'autorisation de se retirer, à la surprise de l'empereur (III, 8, v.1079). A défaut de la solitude profane et quasi pastorale dont elle rêvait, elle manifeste le voeu de se réfugier chez les Vestales, qu'elle rejoindra au dénouement. Selon une expression d'Augustin ${ }^{57}$ souvent reprise à Port-Royal ${ }^{58}$, Junie meurt au monde ("Sans mourir, elle est morte pour lui", ( $\mathrm{V}$, scène dernière, v. 1722) pour renaître dans un temple qui ressemble tant à un monastère de femmes que toute la scène a suscité la raillerie des contemporains. Boursaut persiflait : le dernier acte de Britannicus prenait, disait-il, des airs de "tragédie chrétienne".

D'autres furent si touchés de voir Junie s'aller rendre religieuse de l'ordre de Vesta, qu'ils auraient nommé cet ouvrage une tragédie chrétienne si l'on ne les eût assurés que Vesta ne l'était pas. ${ }^{59}$

Le commentaire se voulait ironique : se pourrait-il qu'il ait touché juste? Christian Biet n'est pas loin de partager ce jugement:

Néron peut exiler ou détruire ses proches, il ne peut aller contre la volonté de Junie de se réfugier chez les Vestales, entendons : auprès de Dieu. Là seulement les « regards effarés » du tyran resteront impuissants. En communion avec le peuple comme une héroïne chrétienne, Junie est protégée par lui dans son désir d'adorer le Seigneur et de se soustraire au Mal. Au Mal représenté sur la scène, s'oppose donc le Bien, hors scène. ${ }^{60}$

La princesse aurait-elle été touchée par la grâce de la conversion ? Son attitude idolâtre, face à la statue de son grand-père, incline à en douter $(\mathrm{V}$, scène dernière, $\mathrm{v}$. 1731-1738). Rien dans le texte, ni chez les historiens, ne suggère qu'elle ait pu croiser le chemin de ces premiers chrétiens que l'empereur persécutera bientôt. Cherchant confusément le vrai Dieu, Junie se précipite au contraire aux pieds des idoles, avant de se réfugier dans une caricature d'abbaye où brûle un feu trompeur, qui se contente de mimer la flamme intérieure de la grâce. On n'imagine pas Junie trouver au fond du temple consolation ni repos, sauf à penser qu'elle a trop de vertus pour n'être pas

prête la main, avec Pierre Nicole, d'Andilly, La Fontaine et Brienne, à une importante anthologie poétique en trois volumes, qui paraîtra en 1670. A la même date, Racine est en affaires avec d'Andilly. "C'était l'heure de la grande réconciliation", insiste le biographe, p. 348.

${ }^{57}$ Sermon CLXX.

${ }^{58}$ Par exemple Epitres de saint Paul aux Galates, éd. I.-L. Le Maistre de Sacy, Bruxelles, Fricx, 1709, tome III, p. 177 : "Il nous faut donc prier Dieu qu'il nous fasse mourir au monde, et que le monde soit pareillement mort pour nous".

${ }^{59}$ Edme Boursault, "Artémise et Poliante", in Jean Racine, Théâtre-Poésie, op. cit., p. 440.

${ }^{60}$ Christian Biet, "Les larmes de Junie", in "Britannicus", Encyclopedia Universalis. 
chrétienne, mais rien ne vient positivement confirmer cette lecture. La retraite choisie par Junie n'est pas sans préfigurer, par son indécidable ambiguïté, celle de Mme de Clèves.

Une lecture de Britannicus qui réduirait la pièce à un simple affrontement des victimes face au bourreau ne rendrait pas justice à la complexité de rapports moraux qui s'étendent nécessairement par-delà les bornes de l'éthique ordinaire, et qui constitue ce qu'on appelait naguère encore le tragique racinien.

\section{Conclusion}

Britannicus illustre trois aspects essentiels de la pensée de Port-Royal. Le "règne du coeur mauvais" ${ }^{\prime}$, la nécessité de fuir le siècle corrupteur, et l'étendue de la misère de l'homme, qui rend vaine et caduc tout espoir en une solution morale purement humaniste telle que la philosophie stoïcienne. Marc Fumaroli avait montré comment les personnages de Phèdre étaient des païens qui se débattaient "en proie à de faux dieux"62. La transcendance ne paraît pas aussi présente dans Britannicus, mais au fond, la situation n'est pas si différente : c'est le même spectacle d'une humanité lamentable qui nous est donné à voir. Les héros en apparence vertueux sont eux aussi victimes de leurs illusions, livrés à leurs passions, habités par des rêves irréalisables, en l'absence d'une grâce qui seule pourrait combler le vide de leur coeur. Ni le souverain Bien des philosophes, ni l'amour galant, ni le culte de Vesta n'apporteront la paix et le bonheur à ces héros déchirés. La vertu des païens n'est qu'une illusion. Certes, Racine est un Ancien, et il prendra farouchement partie en faveur de Boileau lors de la Querelle qui éclatera à la fin des années 1680. Mais, tout pétri qu'il soit de culture gréco-latine, il n'est pas pour autant admirateur de leur morale erronée. Racine éprouve simultanément pour les Anciens une vénération esthétique et une répulsion éthique. Ce regard ambivalent, il peut le devoir aux enseignements de son maître Pierre Nicole, dont Béatrice Guion a montré qu'il entretenait le même rapport équivoque à la culture païenne:

Admiration d'humaniste pour les beautés des textes des anciens, rejet des morales antiques par le moraliste, refus du théologien de reconnaître le caractère méritoire et $a$ fortiori salvifique des vertus des païens, ainsi pourrait-on résumer les sentiments de Nicole à l'égard de l'antiquité païenne. ${ }^{63}$

C'est par rapport à cette ambiguïté que s'éclaire le tragique racinien. Les biographes du dramaturge suggèrent que, en cette fin de la décennie 1660, Jean Racine se rapprochait de ses anciens maîtres. Britannicus laisse voir qu'il n'a jamais oublié leurs leçons.

\footnotetext{
${ }^{61}$ Pour reprendre la formule de Philippe Sellier dans son Pascal et saint Augustin, op. cit.

${ }^{62}$ Marc Fumaroli, "Entre Athènes et Cnossos. Les dieux païens dans Phèdre", Revue d'Histoire littéraire de la France, première partie, janvier-février, 1993, p. 42.

${ }^{63}$ Béatrice Guion, "Nicole et l'Antiquité païenne », dans Pierre Nicole, Chroniques de Port-Royal, 45, 1996, p. 43-61.
} 
